\title{
The study of practical legal cases as an effective method of acquiring the discursive communicative skills of international jurists when learning the professional foreign language (professional French)
}

\section{[L'etude de cas pratiques juridiques comme methode efficace d'acquisition des competences communicatives discursives des juristes internationalistes lors de l'apprentissage de la langue etrangere professionnelle (français professionnel)]}

\author{
Ekaterina A. Samorodova - Mikhail K. Ogorodov - Irina G. Belyaeva - \\ Elena B. Savelyeva
}

DOI: 10.18355/XL.2020.13.01.10

\begin{abstract}
In this article the authors focus on the study of practical legal cases as one of the effective methods in foreign discursive communicative competence training in professional French courses taught by faculties whose specialization is international law. The procedure for resolving a practical case in French requires close coherence and interaction between the pragmatic, linguistic and professional components of the teaching process. This is the main objective of the authors who propose a selection of legal practical cases in order to integrate them into the system of teaching French as a professional based on the following criteria: linguistic and professional. In this case, the authors propose an appropriate algorithm for solving practical cases in professional French courses for students who specialize in international law.
\end{abstract}

Key words: communicative discourse competence, legal question, practical case, professional foreign language; professional French, teaching method, juristinternationalist

Résumé : Dans le présent article les auteurs portent à l'étude de cas pratiques juridiques comme une des méthodes efficaces dans la formation à la compétence communicative discursive étrangère dans les cours de français professionnel dispensés par les facultés dont la spécialisation est le droit international. La procédure de résolution d'un cas pratique en français nécessite une cohérence et une interaction étroites entre les composantes pragmatique, linguistique et professionnelle du processus d'enseignement. C'est l'objectif principal des auteurs qui proposent une sélection de cas pratiques juridiques afin de les intégrer au système de l'enseignement $\mathrm{du}$ français professionnel en s'appuyant sur les critères suivants: linguistique et professionnel. En l'occurrence, les auteurs proposent un algorithme approprié de résolution de cas pratiques dans les cours de français professionnel pour les étudiants qui se spécialisent en droit international.

Mots-clés : compétence discursive communicative, question juridique, cas pratique, langue étrangère professionnelle; français professionnel, méthode d'enseignement, juriste-internationaliste

\section{Introduction}

La dimension de la concurrence dans le monde contemporain se manifeste surtout dans les domaines professionnels supposant une coopération avec les partenaires étrangers. Le développement des processus d'intégration accroît la demande sur le marché du travail pour les spécialistes maîtrisant une langue étrangère au niveau 
professionnel, ou plutôt langue de spécialisation. Ce fait est considéré dans certaines recherches ( Sun, 2013; Kramsch, 2014; Kuznetsova, 2015).

La maîtrise d'une langue étrangère professionnelle implique la capacité de communiquer librement dans ce domaine avec une bonne connaissance du lexique et du style approprié, ainsi que la maîtrise de leur emploi. Une compétence discursive professionnelle étrangère dans les termes du présent article est comprise comme celle qui réunit à la fois les notions de communication verbale et non-verbale pratiquée dans le cadre d'une communication professionnelle. Conformément à cette définition donnée du discours professionnel, l'enseignement de la langue étrangère propose tant la composante linguistique que la composante professionnelle. Cela permet de constater que la formation des compétences professionnelles discursives des apprenants revêtent un caractère interdisciplinaire : d'où vient la nécessité pour le professeur de se mettre en quête de nouvelles méthodes et techniques d'apprentissage de la langue étrangère professionnelle susceptibles d'introduire des connaissances professionnelles indispensables au processus pédagogique. Les particularités de l'élaboration de telles méthodes et pratiques dépendent expressément du caractère de la future spécialisation.

Les langues étrangères font partie intégrante du métier de tout juriste internationaliste. Cela implique en outre des compétences professionnelles discursives spécifiques pour l'activité du futur spécialiste dans le domaine du droit international. Ses compétences professionnelles supposent qu'il puisse intervenir dans le domaine normatif, c'est-àdire dans les discussions, l'élaboration, l'adoption, la modification, l'authentification des actes juridiques, la participation aux négociations et conférences internationales, la résolution des problèmes et situations juridiques, l'exercice de son activité dans les instances judiciaires, la consultation, voir la traduction d'actes juridiques. Ainsi le but de tout enseignement professionnel est de rapprocher au maximum le processus de formation de la future activité professionnelle. Selon les auteurs du présent article la résolution des cas pratiques juridiques est une des techniques et méthodes les plus efficaces de l'enseignement et l'apprentissage de la langue étrangère (le français) professionnelle et de la formation d'une compétence discursive communicative des juristes internationalistes.

\section{Littérature étudiée}

Le 20-ème sciècle a rapporté un nombre considerable de méthodes efficaces de l'enseignement des langues étrangères. On pourrait nommer parmi ces méthodes les plus répandues telles qui appliquent ICT/TIC (Bilyalova,2017; Chen, I-Jung; Chang, Chi-Cheng; Yen, Jung-Chuan, 2012; Fedotova, 2015; Hong, J.-C.; Hwang, M.-Y.; Tai, K.-H.; Lin, P.-H, 2017; Luka, 2018; Kenning,2007; Kogan, Gavrilova, Nesterov, 2018; Mazur, Rzepka, Araki, 2015) et filmes (Vyushkina, 2016), ensuite modélisation en cadre (Ignatkina, 2018), méthode de projet ( Bokut, Serebryantseva, 2016; Nargis, Armelia, 2018), brainstorming (Nechayuk,2017; Unin, Bearing, 2016), jeu de rôles (Kalyuzhnaya, Skorobogatova, Vlasova, 2015; Prikoszovits, 2017), tandem (Pomino, Jenny \& Salom, Daniela,2016; Alberth, Wang, Wang, 2019), lecture étendue (Bernstein, 2017), méthode podcast (Kavaliauskienè, Anusienè, 2009; Masudul Hasan, MD \& Tan, B. H., 2013), associative (Kröger, Kannampuzha, Kaufmann, 2014; Vasyuhnevich, 2016), méthode de coopération (Arsentyeva, Gulk, Kasyanik, 2016; Mikhina, Potrikeeva, 2018; Sharan, Shachar, 1988; Wysocki, 2010), sliding (Antselevich, Smagrinskaya, Malakhov, 2015), méthode de la linguistique contrastive (Durst, 2017), discussion (Shelestova, Zagidullina,2014), dilemme (Kholod, 2018), Jingsaw Reading (Yuhananik, 2018), production théâtrale (Kungurova, Voronina, Dolzhenko, 2014), SCRU|M (Jurado-Navas, Munoz-Luna, 2017), table ronde (Rodomanchenko, 2017), Peer review (Bradley, 2012; Sysoyev, Merzlyakov, 2016 ), mnemotéchnique (Ostrikova, Zheltukhina, Zyubina, Sidorova, 2018; Raugh, 
Schupbach, Atkinson, 1977), Methode de grammaire et de la traduction (Thamarana, 2015), audio-lingual (Bidenko, Shcherbak, 2017), méthode directe (Thamarana, 2015), méthode de lecture de Dr. Wes (Thamarana, 2015), classe renversée (Bidenko, Shcherbak, 2017; Wang, An, Wright,_2018; Tikhonova, Ilduganova, Lukina, 2018 ), CLIL ou EMILE (désigne «l'Enseignement d'une Matière Intégré à une Langue Etrangère,» c'est-à-dire l'enseignement d'une matière autre qu'une langue étrangère (par ex. l'histoire, la géographie, les mathématiques, l'économie, etc.) en langue étrangère [Popova, Vdovina, 2018; San Isidro, X., 2018).

A nos jours, lors de l'enseignement de la langue professionnelle (dans le contexte du présent article du français juridique), l'approche CLIL/EMILE prend du terrain, car il permet de réunir la formation linguistique et professionnelle (juridique,economique. etc) (Vyushkina, 2017), ce qui permettra au diplômé de se sentir libre et confidant dans le discours professionnel en langue étrangère.

Parmi les méthodes efficaces de l'enseignement axées sur les compétences (Fesenko, Fedyaeva, Bestsennaya, 2017; Holubnycha, 2016; Hsu, 2016; Lichtensteiner, 2011) et control (Schaper, Hilkenmeier, 2013: 31) et qui sont les plus largement utilisées dans le monde on pouraitt nommer Case - méthode. En Allemagne également appelée Fallstudie, en France, elle est appellee aussi -méthode des cas.

Cette méthode, ayant principalement trouvé son application dans l'étude du droit (Garvin, 2003) et des disciplines économiques (Liening, Paprotny, 2005), est à present largement utilisée dans l'enseignement et il ne s'agit pas uniquement de la formation supérieure, grandes écoles et universités (Brugger, Kyburz-Graber, 2016: 14), de la formation professionnelle spécialisée (Schenk, Reggelin, Barfus,2006: 105). Ladite méthode est appliquée dans le système de l'enseignement secondaire (Lichtensteiner, 2011). Actuellement Il existe des règles générales spécialement élaborées pour la méthode des cas (Bonz, 2009; Riedl, 2012), on délibère sa place et son rôle dans les méthodes interactives (Riedl, 2012).

Il est nécessaire d'ajouter que la méthode d'étude de cas pratiques est une des méthodes d'enseignement orientées vers la formation des compétences professionnelles. En pratique la méthode d'étude de cas peut également réunir les méthodes telles que les ICT/TIC, le brainstorming, les jeux de rôle, la coopération, la discussion, la prise de décision, l'entreprise de formation, EMILE/CLIL, dont le but principal est de résoudre un problème dans une langue étrangère en employant le discours professionnel.

La méthode d'étude de cas pratiques juridiques ou CPJ (abréviation introduite par auteurs) est un des objets de recherche du présent article, elle est considérée comme une des plus efficaces lorsqu'il s'agit de l'enseignement de la langue professionnelle étrangère (le français dans le contexte de l'article) et de la formation des juristes en général (Jesgarzewski, 2018). Deux termes pourraient être employés dans la terminologie pédagogique pour refléter l'essence de la méthode : une tâche juridique ou un cas pratique, donc un cas tiré de la pratique. Les auteurs de cette étude respectent les deux mais sont plus enclins à utiliser celle de «cas pratique juridique » $(\mathrm{CPJ})$.

Un cas pratique juridique est une situation problématique créée par certaines circonstances nécessitant le recours à des moyens juridiques pour trouver immédiatement une solution au problème créé par ces circonstances. 
La méthode de l'étude de cas pratiques s'est fait voir en tant que méthode pédagogique vers la fin du $\mathrm{XIX}^{\mathrm{e}}$ siècle, lorsque, pour la première fois, sous la direction du doyen de la faculté de droit de Harvard, Christopher Columbus Langdell (Garvin, 2003), dans la formation des juristes outre l'étude des actes juridiques a été incluse la jurisprudence.

$\mathrm{Au}$ cours de cette période, une grande expérience dans la résolution des CPJ lors du processus de formation des juristes a été acquise : on a réussi à créer une typologie des cas pratiques et à établir une méthodologie de leur résolution (ZHalinskij, 2009).

Par conséquent, les enseignants recourent à cette méthode pour enseigner aux étudiants la langue étrangère dans le domaine du droit (international, administration publique, etc.) (Bilova, 2016.; Holubnycha,2016).

Les cas pratiques juridiques didactiques appliqués dans les cours de français professionnel à l'Université des relations internationales de Moscou sont l'objet principal de l'étude du présent article. Lesdits cas pratiques résolus pendant les cours de français et en français servent à l'acquisition des compétences linguistiques (puisqu'il est question de l'activité en français) et professionnelles (puisque c'est le domaine de droit) simultanément. Cette combinaison développe à son tour une compétence discursive communicative nécessaire pour le futur spécialiste.

L'objectif principal de la recherche est de mener une analyse profonde des CPJ éducatifs, d'établir leur classification d'après les critères juridique et linguistique, de dégager leurs types d'après leur méthode de résolution et d'élaborer l'algorithme de résolution des $\mathrm{CPJ}$ en français (langue étrangère) sous les formes écrite et orale.

La pertinence de cette étude repose sur la nécessité d'élaborer de nouvelles techniques et méthodes dans le système d'enseignement du français professionnel (langue étrangère spécialisée), qui à leur tour contribueraient à l'acquisition d'une compétence discursive communicative chez les étudiants des facultés de droit international. Selon les auteurs de l'article, le cas pratique, en tant que méthode de formation, est l'outil le plus efficace utilisé en cours de langue étrangère car il permet de rapprocher le plus possible le processus de formation à l'activité professionnelle future, puisque effectivement la profession d'un juriste internationaliste est inextricablement liée à la résolution de problèmes juridiques (litiges, conflits). Et la particularité d'une telle activité est le fait qu'elle se passe en une langue étrangère. À cet égard, il revient nécessaire d'une sélection nette et exacte des textes des CPJ éducatifs conformément aux critères déterminés.

Les principales méthodes de recherche employées pour cet article sont les suivantes : méthode comparative, méthode d'analyse systématique.

\section{Résultats de la recherche}

Critères de la sélection des cas pratiques juridiques dans les buts didactiques pendant les cours du français professionnel de la spécialité Droit international

La capacité à résoudre des problèmes juridiques en une langue étrangère est une des compétences professionnelles les plus importantes que les juristes se spécialisant en droit international veulent acquérir. Cette affirmation est justifiée par les résultats de 
l'enquête menée auprès des étudiants de $3^{\mathrm{e}}$ et $4^{\mathrm{e}}$ années de la faculté de droit international de l'Université des relations internationales de Moscou. Au total, 150 étudiants ont participé à l'enquête. Chacun a dû choisir sur la liste la compétence qu'il considérait comme la plus importante.

Parmi les compétences professionnelles des juristes internationalistes, ont été dégagées les suivantes (tableau №1) :

Tableau № 1: Compétences professionnelles des juristes. Nombre d'étudiants ayant préféré telle ou telle compétence.

\begin{tabular}{|l|l|}
\hline Compétences professionnelles & Nombre d'étudiants \\
\hline Consultation & 26 \\
\hline Participation à des négociations & 20 \\
\hline Élaboration d'actes juridiques & 22 \\
\hline Intervenir dans les juridictions & 23 \\
\hline $\begin{array}{l}\text { Participation à des conférences } \\
\text { internationales }\end{array}$ & 18 \\
\hline Traduction de textes juridiques & 9 \\
\hline Résolution de cas pratiques juridiques & 32 \\
\hline
\end{tabular}

Résultats de l'enquête présentés sur le graphique 1.

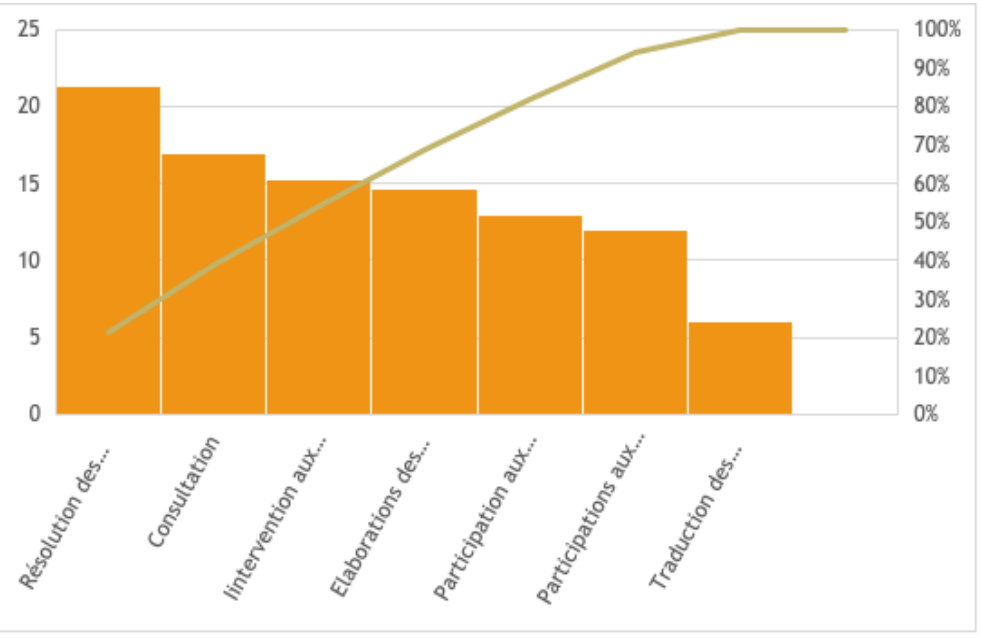

Graphique 1 :

XLinguae, Volume 13 Issue 1, January 2020, ISSN 1337-8384, ISSN 2453-711X 
Participation à des négociations

Participations à des conférences

Intervention dans des juridictions

Traduction de textes juridiques

Résolution de cas pratiques

Élaborations d'actes juridiques

Les résultats de l'enquête montrent que

la résolution de cas pratiques juridiques est la compétence la plus souhaitée que les futurs spécialistes en droit international veulent acquérir : 32 étudiants sur 150 (21,35 $\%)$ ont souligné l'importance du cas pratique.

Les thématiques principales dans le cadre desquelles il est procédé au choix des CPJ pour les étudiants en droit qui apprennent le français relèvent du domaine du droit international public, y compris les notions générales et spéciales. On pourrait en dégager les suivantes:

\section{A. Notions générales}

- $\quad$ Définition du droit international ;

- $\quad$ Sources du droit international ;

- $\quad$ Sujets du droit international ;

- $\quad$ Responsabilité dans le droit ;

- Le système judiciaire international ;

B. Questions (notions) spéciales :

- Droit aérien;

- Droit maritime ;

- Droit humanitaire ;

- $\quad$ Droit de l'environnement ;

- $\quad$ Protection des droits de l'homme ;

- Droit économique.

La jurisprudence de la Cour internationale de Justice, du Tribunal de la mer, (maritime), de la Cour européenne des droits de l'homme, ainsi que les manuels et dossiers didactiques sur le droit international traduits en français servent d'exemple de CPJ aux cours du français professionnel.

Le choix d'un cas pratique est expliqué par les buts concrets, que poursuit le professeur au cours.

Les fonctions dominantes des cas pratiques, celles qui sont inhérentes à tous leurs types, sont l'amélioration de la compétence de maîtriser les nombreux actes juridiques; formation d'une logique juridique cohérente (quelles sont les questions que se pose le juriste, quelles décisions intermédiaires il prend, quels documents, actes juridiques normatifs pour résoudre telle ou telle situation il applique, quels résultats préliminaires peut-il obtenir, quelles seront les conséquences juridiques de sa décision), ainsi que la formation de la compétence d'une opinion argumentée rédigée en français (ou autre langue étrangère) sur des problèmes différents juridiques. 
Afin d'appliquer les CPJ aux cours de la langue française professionnelle on passe à leur classification d'après deux critères :

\section{- $\quad$ Linguistique.}

Selon ce critère il faut prendre en considération le niveau de la maîtrise des compétences linguistiques des étudiants. Conformément au Cadre européen commun de référence pour les langues on propose les textes des cas pratiques selon les niveaux $\mathrm{B} 1 / \mathrm{B} 2 / \mathrm{C} 1$. Les textes des cas pratiques se diffèrent considérablement par la terminologie, par la richesse des structures de grammaire et lexique, aussi par son authenticité. Pour les étudiants ayant un bon niveau de la langue et du droit on propose des textes compliqués authentiques non-adaptés des vrais cas pratiques courants, des différends internationaux qui ont été soumis à la Cour internationale de justice. Pour ceux dont le niveau n'est pas très avancé on élabore des cas pratiques le contenu linguistique desquels correspond au niveau de la langue. Le plus souvent se sont des cas pratiques adaptés. Les exemples sont présentés dans le tableau 2.

- Juridique. résolution.

Selon ce critère tous les cas pratiques sont divisés par types et méthodes de

Selon les types on dégage les cas pratiques :

$\longrightarrow$ D'évaluation et de correction, contribuant à la formation de la position active d'un étudiant ;

De recherche, permettant l'acquisition de nouvelles connaissances et compétences en quête de la résolution d'une situation problématique ;

de recherche et d'analyse permettant à un apprenant de produire des conclusions indépendantes et de prendre des décisions en s'appuyant sur ses connaissances et ses compétences acquises précédemment.

\section{Classifications des CPJ éducatifs appliqués aux cours du français professionnel.}

Effectivement tous les CPJ étudiés en général dans l'enseignement, soit du droit soit de la langue étrangère professionnelle, sont divisés en deux groupes : théorie et pratique.

L'objectif principal des cas juridiques théoriques est d'acquérir des connaissances théoriques dans le domaine de droit. Ladite formation s'effectue durant les conférences, séminaires et colloques de droit international.

Dans le cadre de l'enseignement du français professionnel (une langue étrangère professionnelle) les CPJ orientés vers la pratique sont plus appréciés lorsqu'ils contribuent à la formation des compétences pratiques et connaissances professionnelles communicatives dans le domaine du droit.

Sur le Tableau №2 les auteurs ont présenté la classification des CPJ selon leur forme d'expression, le nombre de participants et les connaissances et savoirs à acquérir.

XLinguae, Volume 13 Issue 1, January 2020, ISSN 1337-8384, ISSN 2453-711X 


\begin{tabular}{|c|c|}
\hline Forme d'expression & $\begin{array}{ll}\text { - } & \text { Écrite } \\
\text { - } & \text { Orale }\end{array}$ \\
\hline Nombre de participants & $\begin{array}{ll}\text { - } & \text { Individuelle } \\
\text { - } & \text { Collective }\end{array}$ \\
\hline $\begin{array}{l}\text { Connaissances et savoirs à } \\
\text { acquérir }\end{array}$ & $\begin{array}{l}\text { - } \quad \text { Art oratoire en français (langue étrangère) } \\
\text { Lettre juridique en français (langue } \\
\text { étrangère) } \\
\text { Recherche de l'information normative, } \\
\text { réglementaire et factuelle } \\
\text { Étude et analyse de l'information } \\
\text { normative, réglementaire et factuelle } \\
\text { - Interprétation des actes juridiques }\end{array}$ \\
\hline
\end{tabular}

Conformément les méthodes de résolution CPJ [46], on distingue 4 types :

- Stated - Problem Method.

- Case-Problem Method

- Case - Study Method

- Stated - Incident Method

Selon Stated - Problem Method toutes les informations nécessaires pour résoudre un CPJ sont prévues dans le texte autant que le problème. De plus tous les problèmes juridiques sont clairement formulés et certaines variantes de résolution correcte figurent.

Les CPJ qui sont résolus par Case - Problem Method contiennent toutes les informations pour les résoudre, les problèmes de droit sont formulés mais il n'y a pas de variantes de résolution.

Case - Study Method présente toutes les informations dans le texte du CPJ mais les problèmes de droit ne sont pas évidents, les questions ne sont pas formulées.

À la différence des autres méthodes Case - Incident Method mentionne plusieurs problèmes de droit dans un CPJ, mais ils ne sont pas évidents; de plus, bien que le texte du cas soit étendu celui-ci ne contient pas assez d'informations pour le résoudre.

La résolution des $\mathrm{CPJ}$ invoque, comme déjà mentionné dans le présent article, une interaction étroite des composants linguistique et juridique de la compétence discursive communicative d'un juriste internationaliste. Dans le Tableau № 3 les auteurs proposent une sélection des cas pratiques conformément le niveau de maîtrise de la langue et du contenu juridique. 


\section{Stated - Problem Method}

Exercice d'évaluation et de correction, B1

Exercice - test :
L'étudiant doit choisir une des
variantes de résolution d'un cas
pratique, argumenter son choix en
s'appuyant sur ses connaissances en
droit et formuler son opinion en
français (langue étrangère)

Exemple : Au cours d'un vol international sur la ligne Paris-Toronto, un avion français appartenant à Air France effectue un atterrissage imprévu à l'aéroport international de New York en raison d'une grave panne d'électricité à bord.

Les fondements juridiques pour effectuer le vol Paris-Toronto sont :

Convention de Chicago de 1944 ;

- Traité bilatéral portant sur le transport aérien entre la France et le Canada ;

- $\quad$ Accord de transit (1 liberté) entre la France et les États-Unis Aucune autorisation préalable d'atterrir au territoire dudit aéroport n'a été reçue.

Question : L'acte commis par un avion français constitue-t-il une violation ? Réponses :

Oui. Tout vol dans l'espace aérien d'un autre État sans autorisation préalable est une violation.

Non. Il s'agit d'un atterrissage forcé, créé par des conditions de la force majeure (qui est imprévisible, irrésistible et échappe au contrôle de l'État).

\section{Case - Problem Method}

Exercice de recherche

B2

\section{Exercice - question : \\ L'étudiant doit répondre en français (une langue étrangère) aux questions déjà formulées dans l'énoncé du cas pratique construisant sa réponse sur les connaissances en droit.}

Exemple : Lors d'une réunion ordinaire de l'Assemblée générale des Nations Unies, le chef de l'État de A., déclarant que le gouvernement de l'État de B. violait les principes de la démocratie en portant une grave atteinte aux droits de l'Homme a proposé de prendre des mesures coercitives visant à renverser le régime existant dans l'Etat de $B$. en recourant aux forces armées si nécessaire. À son tour l'État de B. fait appel au Conseil de sécurité des Nations Unies en lui demandant de traduire l'État de A. devant la cour pour avoir violé un des principes généraux du droit international, notamment celui de non-ingérence dans les affaires intérieures d'un État, ainsi que le principe de l'égalité souveraine des États.

Questions :

1. La déclaration du chef de l'État de A. constitue-t-elle une violation du principe de non-ingérence dans les affaires intérieures d'un État?

2. L'appel de l'État de B. au Conseil de sécurité des Nations Unies est-il raisonnable?

Citez le droit international applicable. 


\begin{tabular}{l|l}
$\begin{array}{l}\text { Case - Study Method } \\
\text { Exercice de recherche et d'analyse } \\
\text { B2-C1 }\end{array}$ & $\begin{array}{l}\text { Exercice à une résolution libre } \\
\text { ouverte : } \\
\text { Le problème du droit est évident dans }\end{array}$ \\
le texte du cas pratique mais les \\
questions ne sont pas formulées. Il \\
revient à l'étudiant de formuler les \\
questions nécessaires et en y répondant \\
en français pour trouver la résolution \\
du cas pratique.
\end{tabular}

Case - Incident Method

Exercice d'évaluation et de correction, de recherche et d'analyse

C1

\section{Exercice à résolution libre ouverte contenant plusieurs problèmes juridiques : \\ L'étudiant relève tous les problèmes du droit dans le cas pratique, formule consécutivement des questions, y répond dans l'ordre de priorité afin de trouver une résolution logique du cas.}

Exemple : J. Cerveau, citoyen de l'État de A., ancien professeur de physique nucléaire et d'ingénierie à l'Université, a été envoyé honorablement à la retraite. Pendant ses longues années passées au bureau de recherche en matière de construction, production et exploitation des vaisseaux spatiaux il s'est livré à des tentatives de concevoir des véhicules spatiaux à faible consommation d'énergie. Son but d'en produire un n'a pas été atteint.

Désormais incapable de poursuivre ses recherches à l'institut, J. Cerveau s'est mis au travail dans son atelier à domicile organisé dans cet objectif.

Les aspirations innovatrices scientifiques de Cerveau ont été soutenues par un brevet pour ses activités de recherche et science.

S'étant rendu compte que les risques possibles de conséquences néfastes d'une telle activité pouvaient se produire au cours de ses recherches et causer des dommages matériaux aux personnes physiques des alentours, Cerveau a pris préalablement les mesures de sécurité technique nécessaire.

Après six mois de travaux acharnés passés dans ledit atelier, Cerveau est parvenu à créer un appareil spatial auquel il a attribué la dénomination de Rêve ». Ledit appareil, lancé par le savant depuis le terrain d'essai, ayant quitté la limite supérieure de l'espace aérien souverain de l'État de A et parcouru plusieurs milliers de kilomètres à l'altitude de $120 \mathrm{~km}$, est tombé dans l'État de B., dans la ville de Boum, ayant causé de graves dommages au musée local « Histoire de Boum ».

- À vous de relever les problèmes de droit et de formuler des questions de droit, Indiquer toutes les conséquences juridiques et sélectionner le droit applicable. 
Le contenu juridique et linguistique correspond aux niveau de langue et des disciplines juridiques étudiées. Ainsi les cas pratiques du niveau B1 dont le but principal est de faire argumenter ou commenter un avis, une opinion qui plus tard passent à un jugement sont proposés aux étudiants de troisième année qui apprennent le français comme première langue étrangère ou aux étudiants de quatrième année apprenant le français comme deuxième langue étrangère et ayant un niveau approprié. En conséquence les $\mathrm{CPJ}$ du niveau $\mathrm{B} 2-\mathrm{C} 1$ sont destinés à des étudiants de quatrième année maîtrisant le français au niveau $\mathrm{B} 2-\mathrm{C} 1$ et la finalité principale de la résolution des CPJ est de rédiger un texte logique et cohérent d'une résolution qui reflète la position de l'étudiant envers le problème de droit en question.

La résolution des $\mathrm{CPJ}$ en français (ou autre langue étrangère) comme méthode efficace dans la formation de la compétence discursive communicative des juristes internationalistes s'avère impossible sans interaction entre ses composants principaux: linguistique et professionnel. Les auteurs de la présente recherche proposent un algorithme de résolution des $\mathrm{CPJ}$ en français dans le domaine du droit international aux cours de français professionnel qui est exposé dans le Tableau № 4.

Tableau № 4.

\begin{tabular}{|c|c|c|c|}
\hline \multicolumn{4}{|c|}{$\begin{array}{l}\text { Algorithme de résolution des cas pratiques juridiques en français dans le } \\
\text { domaine du droit international dans les cours de français professionnel. }\end{array}$} \\
\hline & $\begin{array}{l}\text { Étapes de résolution } \\
\text { d'un cas pratique }\end{array}$ & $\begin{array}{l}\text { Composante } \\
\text { professionnelle }\end{array}$ & $\begin{array}{l}\text { Composante } \\
\text { linguistique }\end{array}$ \\
\hline 1 & $\begin{array}{l}\text { Identification d'une } \\
\text { situation } \\
\text { problématique dans un } \\
\text { CPJ } \\
\text { В предыдущих } \\
\text { таблицах не было } \\
\text { курсива }\end{array}$ & $\begin{array}{l}\text { Analyse et évaluation } \\
\text { dirigées des informations } \\
\text { présentées du point de vue } \\
\text { du droit. Définition de la } \\
\text { branche du droit régissant } \\
\text { ces relations }\end{array}$ & $\begin{array}{l}\text { Étude du texte } \\
\text { authentique de CPJ } \\
\text { (travail ave le } \\
\text { contenu du texte, } \\
\text { traduction des termes } \\
\text { et des constructions, } \\
\text { mise en évidence du } \\
\text { problème principal, } \\
\text { formulation du } \\
\text { problème) }\end{array}$ \\
\hline 2 & $\begin{array}{l}\text { Analyse } \\
\text { circonstances et des } \\
\text { faits du problème } \\
\text { juridique du CPJ }\end{array}$ & $\begin{array}{l}\text { Étude approfondie des faits } \\
\text { et des circonstances des } \\
\text { affaires (détermination des } \\
\text { circonstances pertinentes } \\
\text { pour un examen et une } \\
\text { résolution appropriés de } \\
\text { l'affaire, prise en } \\
\text { considération des obstacles } \\
\text { éventuels à la résolution du } \\
\text { problème, etc.) }\end{array}$ & $\begin{array}{l}\text { Le choix des phrases } \\
\text { clés, phrases dans le } \\
\text { texte, termes relatifs } \\
\text { au problème principal } \\
\text { du CPJ }\end{array}$ \\
\hline 3 & 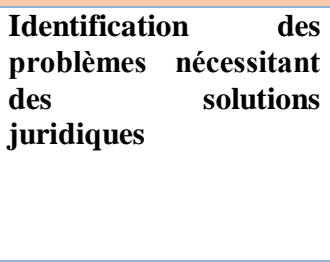 & $\begin{array}{l}\text { Détermination des causes } \\
\text { des circonstances qui ont } \\
\text { conduit à la nécessité } \\
\text { d'utiliser des moyens } \\
\text { juridiques pour résoudre } \\
\text { immédiatement } \\
\text { problème créé par ces }\end{array}$ & $\begin{array}{l}\text { Formulation des } \\
\text { questions qui doivent } \\
\text { être juridiquement } \\
\text { résolues }\end{array}$ \\
\hline
\end{tabular}

XLinguae, Volume 13 Issue 1, January 2020, ISSN 1337-8384, ISSN 2453-711X 


\begin{tabular}{|c|c|c|c|}
\hline & & circonstances & \\
\hline 4 & $\begin{array}{l}\text { Recherche } \\
\text { évaluation des actes } \\
\text { juridiques en termes } \\
\text { de pertinence d'être } \\
\text { appliqué } \\
\text { résoudre CPJ pour }\end{array}$ & $\begin{array}{l}\text { Analyse d'un grand } \\
\text { nombre de documents } \\
\text { juridiques dans le but de } \\
\text { trouver les documents } \\
\text { juridiques nécessaires à la } \\
\text { résolution du CPJ. }\end{array}$ & $\begin{array}{l}\text { Travailler avec des } \\
\text { textes des documents } \\
\text { juridiques en français } \\
\text { (travail sur le contenu } \\
\text { du texte, traduction } \\
\text { des termes et des } \\
\text { structures); }\end{array}$ \\
\hline 5 & $\begin{array}{l}\text { Analyse des solutions } \\
\text { possibles du CPJ }\end{array}$ & $\begin{array}{l}\text { Examen du point de vue de } \\
\text { la réglementation juridique } \\
\text { des différentes résolutions } \\
\text { possibles des problèmes de } \\
\text { droit dans le CPJ }\end{array}$ & $\begin{array}{l}\text { Analyse de la } \\
\text { terminologie } \\
\text { juridique dans les } \\
\text { langues originale et } \\
\text { étrangère (français) }\end{array}$ \\
\hline 6 & $\begin{array}{l}\text { Choix et justification } \\
\text { de la solution optimale }\end{array}$ & $\begin{array}{l}\text { Le choix d'une résolution } \\
\text { juridiquement correcte qui } \\
\text { réponde le mieux aux } \\
\text { conditions énoncées et au } \\
\text { problème de droit dans le } \\
\text { CPJ }\end{array}$ & $\begin{array}{l}\text { Un travail important } \\
\text { avec les textes } \\
\text { juridiques, analyse de } \\
\text { la terminologie au } \\
\text { niveau des deux } \\
\text { langues originale et } \\
\text { étrangère dans le but } \\
\text { d'interpréter } \\
\text { correctement les } \\
\text { informations } \\
\text { juridiques. }\end{array}$ \\
\hline 7 & $\begin{array}{l}\text { Rédaction ou } \\
\text { présentation de la } \\
\text { résolution du CPJ sous } \\
\text { forme orale ou écrite. }\end{array}$ & $\begin{array}{l}\text { Rédaction d'une résolution } \\
\text { bien argumentée (chaque } \\
\text { affirmation donnée dans le } \\
\text { texte qui passe du } \\
\text { problème de droit à une } \\
\text { résolution a besoin } \\
\text { d'arguments qui, à leur } \\
\text { tour, s'appuient sur la } \\
\text { réglementation, la pratique } \\
\text { judiciaire et la doctrine) }\end{array}$ & $\begin{array}{l}\text { Rédaction en français } \\
\text { professionnel d'un } \\
\text { texte cohérent et } \\
\text { logique d'une } \\
\text { résolution du CPJ } \\
\text { bien argumenté, riche } \\
\text { du point de vue des } \\
\text { moyens linguistiques } \\
\text { sous forme écrite ou } \\
\text { orale. }\end{array}$ \\
\hline
\end{tabular}

La forme écrite d'un cas pratique juridique didactique contribue à l'acquisition de la compétence d'exposer logiquement en français (langue étrangère) le processus de résolution d'un problème juridique. Cela rapproche le procès didactique des activités professionnelles des juristes internationalistes telles que la rédaction des règlements, requêtes et arrêts. La forme orale implique essentiellement l'acquisition de compétences telles que l'intervention en public, la persuasion, la coopération, la capacité de défendre sa position, la participation et l'intervention aux débats.

La résolution des CPJ en français dans les cours de français professionnel exige des étudiants de s'orienter rapidement dans les actes normatifs, de connaître exactement les titres des documents en français.

Néanmoins, malgré la présence permanente du droit, l'essentiel dans le processus de résolution d'un CPJ éducatif en français (dans une langue étrangère) n'est pas une résolution correcte du problème qui y est posé du point de vue juridique, 
mais la formation de la compétence discursive communicative basée sur des relations interdisciplinaires, ce qui est fondamental dans la profession d'un juriste internationaliste.

\section{Conclusion}

L'enseignement traditionnel des langues professionnelles étrangères, fondé seulement sur l'enseignement et l'apprentissage approfondis de la grammaire, le lexique et la traduction des termes, ne répond plus aux besoins de la société, puisqu'en raison de l'évolution de la situation économique et politique dans le monde, une connaissance profonde et fondamentale des langues étrangères comme telle n'est plus en valeur. Aujourd'hui il est plus important d'appliquer les connaissances des langues étrangères en pratique dans le domaine professionnel. Cela est dicté par l'approche pragmatique et conduit à la nécessité de créer de nouvelles méthodes et techniques d'enseignement des langues étrangères.

Le cas pratique juridique est une des méthodes qui répondent aux exigences des approches contemporaines dans l'enseignement d'une langue étrangère professionnelle de la spécialisation « droit international ».

La méthode de résolution des CPJ pendant les cours de langue étrangère professionnelle pour les futures juristes internationalistes repose sur les postulats d'une approche communicative dont les attributs principaux sont la pertinence du matériel didactique, les situations communicatives actuelles et la méthode cognitive qui permet aux étudiants d'acquérir d'une manière autonome des connaissances, des savoir-faire afin de trouver, analyser, mettre en ordre les informations reçues, indispensables pour résoudre un problème juridique. Le travail sur les différents types de CPJ, dont le choix est déterminé par l'objectif de la leçon défini par l'enseignant, permet de former et ensuite d'approfondir les compétences de traduction et d'analyse des textes juridiques, l'interprétation et l'argumentation, la rédaction du texte d'une intervention en public ou d'un arrêt judiciaire, ce qui permet aux étudiants de participer pleinement à leurs activités professionnelles dès les études à l'Université.

Grâce aux critères de sélection des CJP énoncés dans cet article, dans le cadre de l'enseignement du français professionnel aux étudiants de licence sur la base des classifications des CPJ appliqués dans le processus éducatif et l'algorithme présenté pour la résolution des CPJ, l'enseignant dispose d'un outil lui permettant d'optimiser son activité pédagogique, d'améliorer les résultats des élèves en fonction de leur niveau, des objectifs de son cours ainsi que de mettre en œuvre la méthode d'étude de cas pratiques dans les livres et manuels spécialisés.

\section{Bibliographic references}

ALBERTH A. - WANG, Y. - WANG, W.Y.C. 2019. Fostering Foreign Language Learning Through a Telecollaborative Social Media-Based Tandem Language Learning Approach. Communications in Computer and Information Science, vol. 10 11, pp. 362-368. Springer, Cham. DOI https://doi.org/10.1007/978-3-030-20798-4_31 ANTSELEVICH, O. - SMAGRINSKAYA, N. - MALAKHOV, B. 2015. Modern educational technologies in non-linguistic university. Scientific enquiry in the contemporary world: theoretical basics and innovative approach research articles. Pedagogik and psychology. vol. 2, pp. 5-8, DOI: 10.15350/L_26/4_2

ARSENTYEVA, X.S. - GULK, E.B. - KASVANIC, P.M. 2016 Applying a Collaborative Learning Technique in $\mathrm{PhD}$ Student Groups with Multinational 
Structure During Foreign Language Studying in Technical University. In Advances in Intelligent Systems and Computing. 19th International Conference on Interactive Collaborative Learning, ICL; Belfast; United Kingdom, vol. 544,

UHOMOIHI J. - GURALNICK D. - AUER M.E. 2017. (eds.); Springer Verlag: Cham, Switzerland, pp. 31-44. DOI: 10.1007/978-3-319-50337-

BERNSTEIN, V.L. 2017. Extracting information as the main component of productive reading // Interactive science. n. 11 (21). pp. 28-31. ISSN 2414-9411. doi:10.21661/r-464693

BIDENKO, L. - SHCHERBAK, H. 2017. Implementing audio-lingual method to teaching Ukrainian as a foreign language at the initial stage. Advanced Education. 3. pp. 23-27. 10.20535/2410-8286.82711.

BILOVA, STEPANKA. 2016. Case Briefs in Legal English Classes. Studies in Logic, Grammar and Rhetoric. pp. 7-20, 45. 10.1515/slgr-2016-0012

BILYALOVA, A. 2017. ICT in Teaching a Foreign Language in High School. Procedia - Social and Behavioral Sciences. 237. pp. 175-181. 10.1016/j.sbspro.2017.02.060. DOI: 10.1016/j.sbspro.2017.02.060

BOKUT, E.L. - SEREBRYANTSEVA, O.G. 2016. Metod proektov i meghpredmetnye svyazi v vuze (na primere predmetov «inostrannyy yazyk» i «psihologicheskoe soprovoghdenie semyi») [project method and interdisciplinary connections in university students teaching ("foreign language" and "family psychological support”)]. Meždunarodnyj naučno-issledovatel'skij žurnal, vol, 2 (44) n. 4, pp. 107-111. http://dx.doi.org/10.18454/IRJ.2016.44.059

BONZ, B. 2009. Methoden der Berufsbildung: ein Lehrbuch. Front Cover. Bernhard Bonz. Hirzel, - Berufsschulunterricht - Unterrichtsmethode - Lehrbuch -

BRADLEY, L. 2012. Peer-reviewing in an intercultural wiki environment-student interaction and reflections. Computers and Composition 34, pp.80-95. DOI: 10.1016/j.compcom.2014.09.008

BRUGGER, P. - KYBURZ-GRABER, R. 2016 Unterrichtssituationen meistern 20 Fallstudien aus der Sekundarstufe II. pp. 248. hep verlag; Auflage: 1., Aufl. (1. Mai 2016)

CHEN, I JUNG CHANG, CHI-CHENG. - YEN, JUNG-CHUAN. 2012. Effects of presentation mode on mobile language learning: A performance efficiency perspective. Australasian Journal of Educational Technology, [S.1.], vol. 28, n. 1, feb. 2012. ISSN 1449-5554. Available online: https://ajet.org.au/index.php/AJET/article/view/887, doi:https://doi.org/10.14742/ajet.887.

DURST, U. 2017. Tekin, Özlem: Grundlagen der Kontrastiven Linguistik in Theorie und Praxis. Informationen Deutsch als Fremdsprache, vol. 41(2-3), pp. 384-386. doi:10.1515/infodaf-2014-2-385

FEDOTOVA, N.L. 2015. On the concept of distance training course "Methods of teaching Russian as a foreign language (RFL): traditions and innovations". Russian Linguistic Bulletin n. 4, pp. 41-43. DOI: https://dx.doi.org/10.18454/RULB.4.05

FESENKO, O.P. - FEDYAEVA, E.V. - BESTSENNAYA, V.V. 2017 Cases in methods of teaching Russian language as a foreign language // Language and Culture. n. 9, pp. 104-117. DOI: $10.17223 / 24109266 / 9 / 12$

GARVIN, DAVID A. 2003 "Making the Case: Professional Education for the World of Practice." Harvard Magazine, pp. 56-65,

HOLUBNYCHA L, O. 2016. Case studies as one of the communication methods of foreign language teaching law students // Legal problems. n. 135. Available online: https://cyberleninka.ru/article/n/case-studies-as-one-of-the-communication-methodsof-foreign-language-teaching-law-students, doi: 10.21564/2414 - 990x.135.78232 UDC 881'243:378 
HONG, J. - C. - HWANG, M.Y. - TAI, K.H. -LIN, P.H. 2017. ntrinsic motivation of Chinese learning in predicting online learning self-efficacy and flow experience relevant to students' learning progress. Computer Assisted Language Learning, vol. 30(6), pp. 552-574 https://doi.org/10.1080/09588221.2017.1329215

HSU, W. 2016. Harvard Business school (HBS) case method to teaching English for business communication, Education and Linguistics Research, vol. 2, n. 2, 98 p., doi:10.5296/elr.v2i2.10192

IGNATKINA, A. 2018. Frame Modeling Method in Teaching and Learning Legal Terminology. Studies in Logic, Grammar and Rhetoric. vol. 53. pp. 81-104. 10.2478/slgr-2018-0005.

JESGARZEWSKI, T. 2018. Fallstudien zum Wirtschaftsprivatrecht, Gabler Verlag, 169 p.,10.1007/978-3-658-19808-4

JURADO NAVAS, A. - MUNOZ-LUNA, R. 2017. Scrum Methodology in Higher Education: Innovation in Teaching, Learning and Assessment. International Journal of Higher Education, pp. 1-18, DOI: https://doi.org/10.5430/ijhe.v6n6p1

KALASHNIKOVA, N. A. 2017. The case study method as a way to develop academic skills and communicative competence of students of an economic university // SISP. №1. Available online: https://cyberleninka.ru/article/n/metod-case-studykak-sposob-razvitiya-akademicheskih-navykov-i-kommunikativnoy-kompetentsiistudentov-ekonomicheskogo-vuza

KALYUZHNAYA, T.V. - SKOROBOGATOVA, E.V. - VLASOVA, A.V. 2015. Business role-play as a means of competence approach implementation in teaching the English language at higher education institutions. Bulletin of Kemerovo State University; vol. 3(2), pp. 52-54. (In Russ.) Available online: https://doi.org/10.21603/2078-8975-2015-2-52-54

KAVALIAUSKIENE, G. - ANUSIENE, L. 2009. English for Specific Purposes: Podcasts for Listening Skills. Santalka. vol. 17. pp. 28-37. 10.3846/1822430X.2009.17.2.28-37.

KENNING, M.M. 2007. ICT and Language Learning. In: ICT and Language Learning. Palgrave Macmillan, London, 225 p. DOI 10.1057/9780230591325

KHOLOD, N.I. 2018. Application of moral dilemmas method for students' communicative competence development in classes of foreign language at higher education institution. Tomsk State Pedagogical University Bulletin. pp. 92-95. 10.23951/1609-624X-2018-7-92-95.

KOGAN, M. - GAVRILOVA, A.V. - NESTEROV, S. 2018. Training Engineering Students for Understanding Special Subjects in English: The Role of the Online Component in the Experimental ESP Course. In Proceedings of the 2018 IV International Conference on Information Technologies in Engineering Education, Moscow, Russia, pp. 23-26, October 2018; pp. 16.10.1109/INFORINO.2018.8581837

KRAMSCH, C. 2014. Teaching Foreign Languages in an Era of Globalisation: Introduction. Modern Language Journal, vol. 98(1), pp. 296-311. doi:10.1111/j.15404781.2014.12057.x

KRÖGER, B.J. - KANNAMPUZHA, J. -KAUFMANN, E. 2014. Associative learning and self-organization as basic principles for simulating speech acquisition,speech production, and speech perception. EPJ Nonlinear Biomedical Physics, Available online: http://www.epjnonlinearbiomedphys.com/content/2/1/2., doi:10.1140/epjnbp15

KUNGUROVA, I.M. - VORONINA, E.V. - DOLZHENKO S.G. 2014. Art technologies in the formation of innovative pedagogical activity among students (on the example of teaching the discipline "Technologies and methods of teaching foreign

XLinguae, Volume 13 Issue 1, January 2020, ISSN 1337-8384, ISSN 2453-711X 
languages"). Online Journal "SCIENCE", vol. 6. pp. 111-115, Available online: http://naukovedenie.ru/PDF/31PVN614.pdf

KUZNETSOVA, E.M. 2015. Evolution of Foreign Language Teaching Methods. Mediterranean Journal of Social Sciences. vol. 6, n 6, S1 November 2015, pp.246-253 DOI: 10.5901/mjss. 2015.v6n6s1.

LICHTENSTEINER, A. 2011. Kompetenzorientierung im Unterricht unter Anwendung der Unterrichtsmethode Fallstudie / Lichtensteiner Astrid , Graz.Univ.Dipl-Arb., 74 p. Open Access. urn:nbn:at:at-ubg:1-27965

LIENING A. - PAPROTNY, C. 2005. Fallstudienarbeit in der Ökonomischen Bildung.Dortmunder Beiträge zur ökonomischen Bildung. Diskussionsbeitrag, n. 8 Dezember, Univ., Wirtschafts- und Sozialwiss. Fak., 5 p. Available online: http://www.wiso.uni-dortmund.de/wd/

LUKA, I. 2018. Summative evaluation of online language learning course efficiency for students studying tourism and hospitality management", Quality Assurance in Education. vol. 26, n. 4, pp. 446-465, https://doi.org/10.1108/QAE-04-2018-0051

MASUDUL HASAN, MD. - TAN, B.H. 2013. Podcast Applications in Language Learning: A Review of Recent Studies. English Language Teaching. 6. 10.5539/elt.v6n2p128.

MAZUR, M. - RZEPKA, R. - ARAKI, K. 2015. Teaching Words in Context: CodeSwitching Method for English and Japanese Vocabulary Acquisition Systems. Human Language Technology. Challenges for Computer Science and Linguistics 7th Language and Technology Conference, LTC Poznań, Poland, November 27-29, 2015, Revised Selected Papers. Lecture Notes in Artificial Intelligence (LNAI), Springer, https://doi.org/10.1007/978-3-319-93782-3_24

MIKHINA, OLGA. - POTRIKEEVA, ELENA. 2018. On the way of the search of new methods in teaching foreign languages (analysis of domestic and foreign experience). Perspectives of science and education. vol. 36. pp. 155-163. 10.32744/PSE.2018.6.17.?

NARGIS, NARGIS - ARMELIA, L. 2018. Optimizing EFL learners' communicative competence through short movie project. Asian EFL Journal. vol. 20, n. 5, pp. 201208.

NECHAYUK I.A. 2017. Active methods as optimization model for teaching English for special purposes. Interactive science, n. 2(12), pp. 96-99. doi:10.21661/r-117837

OSTRIKOVA, G.N. - ZHELTUKHINA, M.R - ZYUBINA, I.A. - SIDOROVA I.G. 2018. Learning Via Visualization at the Present Stage of Teaching a Foreign Language. Astra Salvensis, VI n. 1, pp. 601-607.

PAHL, J.P. 2007. Ausbildungs- und Unterrichtsverfahren. Ein Kompendium für den Lernbereich Arbeit und Technik. Bertelsmann Verlag, 108 p.

POMINO, JENNY - SALOM, DANIELA. 2016. Integrating E-tandem in Higher Education. Procedia - Social and Behavioral Sciences, vol. 228. pp. 668-673. 10.1016/j.sbspro.2016.07.102.

POPOVA, N.V. - VDOVINA, E.K. 2018. Constraints of Communicative Approach to Language Teaching in Russian Tertiary Education. The European Proceedings of Social \& Behavioural Sciences EpSBS. 18th PCSF - Professional Culture of the Specialist of the Future. 28-30 November 2018. Edited by: Prof. Valeria Chernyavskaya and Prof. Holger Kuße. Peter the Great St. Petersburg Polytechnic University. Published by the Future Academy, vol. LI, pp. 1325-1336. DOI: 10.15405 / epsbs.2018.12.02.142

PRIKOSZOVITS, M. 2017. Auf dem Prüfstand - Wie berufsbezogen und praktikabel sind moderne handlungsorientierte DaF-Unterrichtsaktivitäten? In: Magazin (Andalusischer Germanistenverband), pp. 34-45, DOI: http://dx.doi.org/10.12795/magazin.2017.i25.04 
RAUGH, M.R. - SCHUPBACH, R.D. - ATKINSON, R.C. 1977. Teaching a large Russian language vocabulary by the mnemonic keyword method. Instr. Sci., 6, pp. 199-221. https://doi.org/10.1007/BF00120656

RIEDL, A. 2012. Interaktive Lehr-Lern-Methoden: Fallstudie, Rollenspiel, Planspiel, Zukunftswerkstatt und Szenario. In: Larissa Korneeva (Hrsg.): Aktuelle Fragen des Fremdsprachenunterrichts, der interkulturellen Kommunikation und der Fachrichtung Übersetzung an der Hochschule. Sammlung von Beiträgen zur internationalen wissenschaftlich-praktischen Konferenz. Jekaterinburg: UrFU (Uraler Föderale Universität), pp. 104-114, (ISBN 978-5-321-02223-8)

RODOMANCHENKO, A. 2017. Roundtable Discussion in Language Teaching: Assessing Subject Knowledge and Language Skills. Journal of Language and Education, vol. 3(4), pp. 44-51, https://doi.org/10.17323/2411-7390-2017-3-4-44-51

SAN ISIDRO, X. 2018. Innovations and Challenges in CLIL Implementation in Europe, Theory Into Practice, 57:3, pp.185-195, DOI: 10.1080/00405841. 2018. 1484038

SCHAPER, N. - HILKENMEIER, F. 2013. Umsetzungshilfen für kompetenzorientiertes Prüfen. HRK-Zusatzgutachten ausgearbeitet für die HRK von Niclas Schaper und Frederic Hilkenmeier unter Mitarbeit von Elena Bender, 133 p. Available online: http://www.hrk-nexus.de/fileadmin/redaktion/hrk-nexus/07Downloads/07-03-Material/zusatzgutachten.pdf.

SCHENK, M. - REGGELIN, T. - BARFUS, K. 2006. Innovative Lehrmethoden in der universitären und außeruniversitären logistischen Aus- und Weiterbildung. In: Engelhardt-Nowitzki C. (eds) Ausbildung in der Logistik. DUV, pp. 105-118. DOI: https://doi.org/10.1007/978-3-8350-9402-4_8

SHARAN, S. - SHACHAR, H. 1988. Language and Learning in the Cooperative Classroom. Springer-Verlag New York. 176 p. DOI 10.1007/978-1-4612-3860-7

SHELESTOVA, O.V. - ZAGIDULLINA, A.I. 2014. Application of the discussion method for the formation of communication skills in foreign language lessons. Minbar. Islamic Studies, vol. 7(2), pp. 183-193. https://doi.org/10.31162/2618-95692014-7-2-183-193

SUN, J. 2013. Globalization and Language Teaching and Learning in China. International Journal on Integrating Technology in Education. 2. Pp. 35-42. 10.5121/ijite.2013.2404.

SYSOYEV, P.V. - MERZLYAKOV, K.A. 2016. Methods of teaching international relations students writing skills using peer review// Language and culture. vol. 2, n. 34. pp. 195-206.

THAMARANA, S. 2015. A Comparative Study of Various English Language Teaching Methods, Approaches. 10.13140/RG.2.1.4026.5046.

TIKHONOVA, N.V. - ILDUGANOVA, G.M. - LUKINA, M.S. 2018. Implemented Teaching Methods Based on Interactive Learning Process in Order to Increase the Ability of Learning Foreign Language. J. Soc. Sci. Res. vol. 5, pp.473-478.

UNIN, N. - BEARING, P. 2016. Brainstorming as a Way to Approach Studentcentered Learning in the ESL Classroom. Procedia - Social and Behavioral Sciences. n. 224, pp. 605-612, 10.1016/j.sbspro.2016.05.450.

VASYUHNEVICH, I.A. 2016. The associative approach in the study of Latinmedical and pharmaceutical terminology. Sci. Alm. n.7-1(21). pp. 216-219. DOI:10.17117/na.2016.07.01.216

VYUSHKINA, E. 2016. Legal English through movies development of professional communicative competence // Studies in Logic, Grammar and Rhetoric. vol. 45, n. 1, pp. 253-263, DOI: $10.1515 /$ slgr-2016-0027

XLinguae, Volume 13 Issue 1, January 2020, ISSN 1337-8384, ISSN 2453-711X 
VYUSHKINA, E.G. 2017. CLIL Approach to Legal English Courses:Analysis of Practice and Experience // Sustainable Multilingualism. Biannual Scientific Journal. vol. 10/2017, pp. 136-149, DOI: http://dx.doi.org/10.1515/sm-2017-0007

WANG, J. - AN, N. - WRIGHT, C. 2018. Enhancing beginner learners' oral proficiency in a flipped Chinese foreign language classroom, Computer Assisted Language Learning, 31:5-6, pp. 490-521, DOI: 10.1080/09588221.2017.1417872 WYSOCKI, K. 2010. Kooperatives Lernen im Englischunterricht: Die Kommunikationsfähigkeit aktiv fördern-Vom ersten Schuljahr an/Karolina Wysocki. Mit einem Vorw. von Ruth Kirchmann; Neue-Dt.-Schule-Verl.-Ges.: Essen, Germany, 175 p. ISBN 13 978-3-87964-314-1.

YUHANANIK, Y. 2018. Using jigsaw model to improve reading comprehension of the ninth graders of smpn 1 karangploso. Ijoltl: indonesian journal of language teaching and linguistics. 3. 51. 10.30957/IJOLTL.V3I1.404.

ZHALINSKIJ, A.E. 2009. Vvedenie v special'nost' "YUrisprudenciya". Professional activity of lawyers. - 2-edition. - "Prospekt" , 362 p.

Words: 6868

Characters: 50379 (27,99 standard pages)

Assistant Professor Ekaterina A. Samorodova, Phd

French Language Department

Moscow State Institute of International Relations

(MGIMO University)

119454, Moscow, Prospekt Vernadskogo, 76

Russia

ORCID ID: 0000-0002-0702-0253

samorodova.ekaterina.78@mail.ru

Assistant Professor Mikhaïl K. Ogorodov, Phd

French Language Department

Moscow State Institute of International Relations

(MGIMO University)

119454, Moscow, Prospekt Vernadskogo, 76

Russia

ORCID ID: 0000-0002-6140-0513

mkogo@mgimo.ru

Irina G. Belyaeva, Phd

German Language Department

Moscow State Institute of International Relations

(MGIMO University)

119454, Moscow, Prospekt Vernadskogo, 76

Russia

ORCID ID: 0000-0002-7083-3564

irinatimakova@mail.ru

Assistant professor Elena B. Savelyeva, Phd

Romano-Germanic Philology Department

State University of Humanities and Technology

142611, 22 Zelenaya St., Orekhovo-Zuevo, Moscow region,

Russia

ORCID ID: 0000-0002-5278-9408

rektorat@ggtu.ru 